Courrier du Centre international Blaise Pascal

$24 \mid 2002$

Varia

\title{
Aristote et Pascal
}

Hélène Michon

\section{OpenEdition}

Journals

Édition électronique

URL : http://journals.openedition.org/ccibp/533

DOI : $10.4000 /$ ccibp.533

ISSN : 2493-7460

\section{Éditeur}

Centre international Blaise Pascal

\section{Édition imprimée}

Date de publication : 21 novembre 2002

Pagination : 8-25

ISBN : 2-84516-215-4

ISSN : 0249-6674

Référence électronique

Hélène Michon, «Aristote et Pascal », Courrier du Centre international Blaise Pascal [En ligne], 24 | 2002, mis en ligne le 02 décembre 2015, consulté le 01 mai 2019. URL : http://journals.openedition.org/ ccibp/533 ; DOI : 10.4000/ccibp.533

Ce document a été généré automatiquement le 1 mai 2019.

Centre international Blaise Pascal 


\title{
Aristote et Pascal
}

\author{
Hélène Michon
}

1 La seule mention qui est faite d'Aristote dans les Pensées est celle du fragment 457 :

On ne s'imagine Platon et Aristote qu'avec de grandes robes de pédants. C'étaient des gens honnêtes et comme les autres, riants avec leurs amis. Et quand ils se sont divertis à faire leurs Lois et leurs Politiques, ils l'ont fait en se jouant. (fr. 457)

2 Occurrence qui nous laisse à penser que si Pascal avait dû intervenir dans la querelle entre les Anciens et les Modernes, il l'eût certainement fait en épousant le parti des seconds. Pourtant cette unique référence, amicale et irrévérencieuse, au Stagirite, ne doit pas nous laisser ignorer les liens réels qui unissent l'auteur des Pensées à celui de l'Éthique à Nicomaque. La filiation que nous croyons discerner est, en effet, implicite parce qu'inconsciente : Pascal n'a pas lu Aristote, mais nombre de concepts clefs utilisés par ces deux auteurs se retrouvent formulés de même façon. C'est dire combien le XVII ${ }^{\mathrm{e}}$ siècle véhicule un aristotélisme latent et combien se vérifie, cette fois-ci non plus à l'égard des Pères de l'Église, mais à celui du Prince des philosophes, cette assertion de Sacy :

M. Pascal est extrêmement estimable en ce que, n'ayant point lu les Pères de l'Église, il avait de lui-même, par la pénétration de son esprit, trouvé les mêmes vérités qu'ils avaient trouvées ${ }^{1}$.

3 Sur d'autres concepts, en revanche, la rupture est totale : nous aurons l'occasion de le souligner.

\section{I. - La Logique}

4 La logique sera notre premier champ d'exploration ${ }^{2}$. Il est usuel de qualifier de révolutionnaire l'ouvrage d'Arnauld et Nicole La Logique ou l'art de penser, il l'est moins de porter attention à la remarque que cet ouvrage contient, dans l'introduction du Premier Discours :

Celui qui a travaillé à cet ouvrage a tiré quelques réflexions nouvelles d'un petit écrit non imprimé qui avait été fait par feu Monsieur Pascal ${ }^{3}$,

laissant à penser que le texte qui prépare et entame la scission avec la logique aristotélicienne est bien un très court opuscule de Pascal intitulé De l'esprit géométrique, 
dans lequel celui-ci jette les fondements de la logique, reprise ensuite à Port-Royal ${ }^{4}$. De fait, cet écrit pascalien marque une nouvelle étape, dans la mesure où il présente un critère de référence totalement absent de l'Organon: celui de l'ordre géométrique. Le choix de la méthode, souci proprement cartésien, est abordé par Pascal dès les premières lignes de son ouvrage :

Et je n'ai pour [démontrer les vérités déjà trouvées] qu’à expliquer la méthode que la géométrie y observe $\mathrm{e}^{5}$.

5 Cette référence à la géométrie prouve que Pascal élabore ici une logique véritablement nouvelle : une logique mathématique, fondée sur une axiomatique. Cependant, Pascal ne demande pas tant à la géométrie des règles méthodologiques comme Descartes, que des règles logiques qui sont aussi des règles critiques. La logique aristotélicienne vient prendre appui sur un premier principe : l'être fonde toujours la connaissance, et cette antériorité est une antériorité à la fois logique et chronologique. Aristote déclare impossibles, dans les Seconds Analytiques, les deux ordres suivants :

Savoir le pourquoi avant le fait ; (...) connaître ce qu'est une chose quand on ignore si elle existe ${ }^{6}$.

Cette connaissance de l'être se trouve exprimée par le biais du syllogisme ; à travers lui, le Stagirite tente de conférer au discours le même caractère de nécessité que celui de l'être. Aussi procède-t-il à l'analyse suivante : toute déclaration implique un sujet et un verbe, lequel a pour rôle d'indiquer qu'une chose est attribuée à une autre; le verbe permet ainsi de poser des relations attributives et des relations d'immanence entre les deux termes: le sujet et le prédicat-un verbe pouvant toujours se décomposer en copule + prédicat. L'attribution fonctionne comme le nerf de toute proposition logique et fonde une logique basée sur l'inclusion. Elle est également le point d'ancrage de la métaphysique aristotélicienne, dans la mesure où, pour celle-ci, tout être se trouve composé d'une substance et de plusieurs accidents: la proposition logique consiste à attribuer des prédicats au sujet - soit des accidents à la substance, laquelle ne peut jamais être attribut. La distinction logique entre sujet et prédicat est très exactement, pour Aristote, l'analogue de la distinction métaphysique entre substance et accident: la logique est ainsi tributaire de la nature même de l'être. De fait, il souligne, dans le même ouvrage, qu'à tout enseignement donné ou reçu par la voie du raisonnement, préexiste une double connaissance : celle de l'existence de la chose et celle de la signification du mot employé. Ainsi, la logique consiste pour le Stagirite dans une analyse de l'expression, tenue pour correspondante de catégories logiques, qui sont également ontologiques. Or, Pascal, dans la recherche de la vérité, considère le chemin inverse :

On doit toujours définir les choses avant que de chercher si elles sont possibles ou non?.

7 C'est par l'analyse de la notion de définition que nous pouvons comprendre le pourquoi d'un tel renversement.

8 En effet, la définition, point de jonction entre l'être et le mot, s'élabore de manière bien différente chez Aristote et chez Pascal. Pour Aristote, la définition diffère du concept en ce que ce dernier est le fait du voũ, c'est-à-dire de la partie intuitive de l'intelligence, la

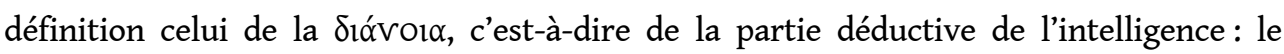
concept est une perception intellectuelle de l'essence - ici encore apparaît l'étroite dépendance de la connaissance à l'être dans la conception aristotélicienne où l'on ne peut connaître ce qui n'est pas - la définition, elle, ne fait pas qu'appréhender l'essence, elle l'explique. Aristote distingue ainsi quatre sortes de définitions qu'il regroupe de la façon 
suivante: d'une part, la définition comme discours expliquant ce que signifie le nom, " discours purement nominal ${ }^{8}$ »; d'autre part, la définition comme quasi démonstration de l'essence dans la mesure où elle prouve; cette deuxième sorte de définition peut être soit un discours indémontrable de l'essence, soit un syllogisme de l'essence ne différant de la démonstration que par la position des termes, soit la conclusion de la démonstration de l'essence.

Pour ce faire, la définition distingue toujours deux éléments: le genre, commun à plusieurs espèces, et les différences non accidentelles par lesquelles ces espèces se différencient entre elles.

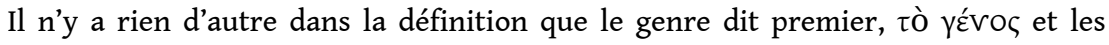

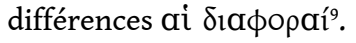

Par exemple, dans la Métaphysique, Aristote définit l'homme comme suit: un animal (le genre), raisonnable (la différence spécifique). La définition est un travail pour cerner l'essence car elle doit prendre les prédicats contenus dans l'essence et les ranger dans leur ordre. C'est pourquoi il n'existe de définition que des substances: celle-ci relève comme telle de la science discursive. Pour souligner le point, Aristote utilise un contreexemple: si on ne les range pas dans leur ordre, on obtient une énumération qui ne possède aucune caractéristique de la définition :

L'homme est un animal pédestre, bipède, sans ailes ${ }^{10}$.

Or, c'est cet exemple que Pascal reprend dans De l'esprit géométrique pour critiquer la conception aristotélicienne de la définition :

Quelle nécessité y a-t-il d'expliquer ce qu'on entend par le mot « homme » ? Ne saiton pas assez quelle est la chose qu'on veut désigner par ce terme ? Et quel avantage pensait nous procurer Platon, en disant que c'était un animal à deux jambes sans plumes $^{11}$ ?

Outre qu'effectivement, présentée ainsi, la définition n'offre plus aucun intérêt: la différence exhibée "à deux jambes, sans plumes », est loin d'être spécifique, - comme le souligne l'auteur des Pensées, à perdre ses jambes, l'homme n'en est pas moins homme -, la critique qu'opère ici Pascal va plus loin : il ne s'agit pas tant en effet de dénoncer une définition douteuse ou, comme le fait Aristote, de souligner que les différences données ne sont pas spécifiques et sont donc inefficaces, mais plutôt de récuser le rôle même accordé à la définition, celui d'expliquer l'essence de la chose. Pascal ne retient, des définitions aristotéliciennes, que la définition nominale. La définition ne fonctionne plus précisément comme point d'ancrage entre le domaine logique et le domaine métaphysique car la définition, dans l'optique pascalienne, ne peut prétendre atteindre l'être ${ }^{12}$. La trilogie « chose, concept, mot » parfaitement hiérarchisée chez Aristote dans la mesure où, de la chose l'esprit abstrait le concept, que le mot signifie de façon parfaitement adéquate, devient chez Pascal dualité : celle de la chose et de son nom, dualité que nous retrouvons dans l'opposition entre définition de nom et définition de chose. La définition a dès lors pour rôle « de désigner les choses que l'on nomme, et non pas [d']en montrer la nature ${ }^{13}$ ", de donner un nom à une idée.

13 La définition possède donc une fonction de désignation, en cela elle exclut toute visée explicative ; c'est pourquoi, comme le souligne Pascal dans sa lettre à Le Pailleur, « on peut aussi bien définir une chose impossible qu'une véritable ${ }^{14}$ ", fait impossible en logique aristotélicienne. Dès lors, le mot, neutre au départ, prend le sens voulu par celui qui l'emploie. Toute autre définition qui viserait à expliquer la nature de la chose désignée, appelée aussi définition de chose, n'est qu'improprement qualifiée de 
définition ; elle est bien plutôt une proposition géométrique, qui demande à être prouvée dans la mesure où elle établit une identité entre le terme compris en son sens ordinaire et la nouvelle définition. Le point commun à Aristote et à Pascal reste la définition nominale ; Pascal fait abstraction de la définition comme travail pour cerner l'essence et en vient directement à la science démonstrative. Pour lui, une définition indémontrable est un non-sens; or, c'est très précisément ainsi qu'Aristote qualifie les principes des démonstrations ${ }^{15}$. la méthode :

... conduire par ordre mes pensées, en commençant par les choses les plus simples et les plus aisées à connaître, pour monter peu à peu comme par degrés, jusqu'à la connaissance des plus composées ${ }^{16}$,

Pascal procède à une véritable atomisation du langage. Cette décomposition est le fait d'un ordre géométrique qui consiste à «n'employer aucun terme dont on n'eût auparavant expliqué nettement le sens ${ }^{17}$ ». En effet, il n'existe pas d'accord universel sur un terme mais uniquement sur le rapport entre un terme et une chose; il faut ainsi, règle d'or en logique pascalienne, substituer toujours la définition au défini, lequel n'est qu'un simple mot. C'est ici que la recherche de l'univocité des termes prend toute sa valeur car elle est intrinsèquement liée à cette conception de la définition comme définition nominale. Pour Pascal, le langage n'est pas simplement un outil au service de la logique, comme il peut l'être pour Aristote, mais le lieu même où s'élabore toute logique. Pour lui, un problème de logique est, avant tout, un problème d'utilisation des termes, qui se résout par une juste utilisation des mots; c'est, en effet, à partir de l'organisation des mots que se pose le problème de la signification. Le fragment 575 des Pensées le souligne :

Et comme si les mêmes pensées ne formaient pas un autre corps de discours par une disposition différente, aussi bien que les mêmes mots forment d'autres pensées par leur différente disposition.

Nous voyons ainsi quel fossé, par le biais de la conception du langage, s'est creusé entre Aristote et Pascal. La logique pascalienne tente, dès lors, de rendre le plus adéquat possible le langage à la pensée humaine - finalité que nous retrouvons bien dans cet ouvrage d'Arnauld et Nicole La Logique ou l'art de penser -, et c'est en cela qu'elle constitue une rupture. À une grammaire fondée sur une analyse de l'expression - la grammaire aristotélicienne - tenue pour correspondante de catégories logiques, succède une grammaire qui se fonde sur une analyse des formes du contenu pour fixer des formes d'expression, la future grammaire de Port-Royal. Le langage se doit, dès lors, de posséder les deux caractéristiques suivantes : il doit être non contradictoire, et les axiomes mis en place doivent pouvoir être utilisés pour toutes les démonstrations : caractéristiques qui, en termes modernes, se nomment consistance et complétude du système et sont propres à la logique symbolique. Pascal fonde ainsi, dans cet opuscule, la première logique axiomatique.

17 Est-ce à dire que Pascal introduit une logique entièrement nouvelle? La présence dans ses ouvrages des termes de principe, évidence ou indémontrable nous interdit de l'affirmer. Une lecture attentive, tant de son opuscule que de son apologie, nous permet en effet de retrouver, exprimées de façon implicite ou explicite, nombre de notions aristotéliciennes. La première se trouve être précisément celle de principe, entendu comme "vérité première ». La notion de principe, chez Pascal comme chez Aristote, empêche une éventuelle régression à l'infini qui consisterait à vouloir démontrer les principes qui 
jouent eux-mêmes le rôle de fondement de la démonstration. Le Stagirite, dans Les Seconds analytiques, a déjà critiqué cette conception d'une démonstration circulaire. Le principe est une vérité dont l'existence est impossible à démontrer; il appartient, comme le dit Pascal, à ces premières vérités connues qui « doivent être accordées et non prouvées ${ }^{18}$ ». Premier logiquement et chronologiquement, il permet à la démonstration de se développer, mais n'entre pas dans son champ d'application. Par rapport à la démonstration, il fonctionne comme un point de départ. Pascal affirme bien, en effet, que la géométrie ne démontre pas toutes choses pour cette seule raison que cela nous est impossible: l'existence des principes est une conséquence de la limitation de notre faculté de connaître, elle est liée à une "impuissance naturelle et immuable ${ }^{19}$ " - et comme telle, elle constitue un élément important de la critique pascalienne de la raison. De même, pour Aristote, aucune proposition ne serait démontrable s'il n'existait pas de principe premier qui, en tant que tel, doive être non point démontré mais bien posé.

La signification du nom se pose, l'existence des principes se pose, l'existence du reste se démontre ${ }^{20}$.

Ces vérités premières sont des définitions indémontrables. Dès lors, il n'existe pas de science absolue ; tout raisonnement nécessite ce point d'appui qu'est le principe ; pour l'un comme pour l'autre, la raison ne possède qu'une perfection relative, non « accomplie ». L'apologiste élabore ainsi une logique qui, à l'image de celle d'Aristote, fait une large place au bon sens, compris comme la juste prise de conscience des capacités de la nature humaine. Et si Pascal note dans l'opuscule De l'esprit géométrique, que :

l'ordre, le plus parfait entre les hommes, consiste non pas à tout définir ou à tout démontrer, ni aussi à ne rien définir ou à ne rien démontrer, mais à se tenir dans ce milieu de ne point définir les choses claires et entendues de tous les hommes, et de définir toutes les autres ${ }^{21}$.

Aristote avait affirmé avant lui :

C'est de l'ignorance de ne pas distinguer ce qui a besoin de démonstration et ce qui n'en a pas besoin ${ }^{22}$.

Cette connaissance s'enracine donc bien dans une capacité de discerner le degré de perfection correspondant à la nature humaine, degré de perfection, dès lors, relatif. La connaissance de l'ordre humain, pour Pascal, est très exactement ce qu'Aristote appelle la sagesse dans l'Éthique à Nicomaque: elle consiste non seulement à connaître les conclusions découlant des principes mais encore à posséder la vérité sur les principes eux-mêmes ${ }^{23}$. Sur ce point encore, Pascal demeure bien proche d'Aristote.

21 Considérons maintenant quelle peut être l'origine de ces principes. Pascal opère une première distinction fondamentale; pour lui, l'origine de nos connaissances peut être double et provenir soit de la démonstration, du "discours" ainsi qu'il l'affirme dans l'opuscule déjà cité, soit de la lumière naturelle et parle alors de "vérités naturelles ". L'ordre géométrique est ainsi dit parfait, dans la mesure où il s'appuie tant sur l'une que sur l'autre: «la nature le soutenant au défaut du discours ${ }^{24}$ ». Les vérités premières ou principes relèvent, elles, non de l'ordre discursif mais de cette lumière naturelle. L'impuissance de la raison, même en géométrie, éclate ainsi, de façon manifeste, face à la double infinité des principes et des propositions ; la force propre de la raison est, en effet, médiatrice ${ }^{25}$ : elle établit des liaisons nécessaires entre ces principes et les conséquences et vice versa, à l'infini, mais elle n'est jamais elle-même une faculté de principe. Pascal précise encore davantage sa pensée dans le fragment 142 de son apologie, en distinguant la raison - qui produit les raisonnements - du cœur ; il affirme que c'est « du cœur que 
nous connaissons les premiers principes ». Il l'unit également à une autre notion : celle de l'instinct.

Car la connaissance des premiers principes (...) est aussi ferme qu'aucune de celles que nos raisonnements nous donnent. Et c'est sur ces connaissances du cœur et de l'instinct qu'il faut que la raison s'appuie et qu'elle y fonde tout son discours. (fr. 142)

Le point commun à ces trois notions - lumière naturelle, instinct et cœur - vient de leur opposition à la raison, entendons la raison discursive, la ratio. Ces termes désignent cependant des réalités différentes: le cœur est la faculté proprement dite, dont la connaissance est naturelle et qui procède de manière intuitive ou instinctive. Mais le plus intéressant à noter ici est que les trois tendent à désigner une même réalité : une faculté de connaître non médiate mais immédiate, aussi Pascal les a-t-il unies dans le fragment 187 des Pensées ${ }^{26}$.

Aristote dans les Seconds Analytiques explique que l'entendement possède deux habitus qui peuvent être vrais ou faux - l'opinion et le raisonnement-, et deux habitus qui sont toujours vrais : la science et l'intuition ${ }^{27}$. Or, il ne saurait exister de science des principes, parce que la science procède par raisonnements et que les principes sont plus connaissables que les démonstrations; dès lors, ce ne peut être que l'intuition, et non la science, qui appréhende les principes. L'origine de nos premiers principes ne peut être attribuée à la partie déductive de notre intelligence, - la raison pour Aristote, l'esprit de géométrie et l'esprit de finesse pour Pascal ${ }^{28}$. Pouvons-nous en conclure maintenant que l'intuition aristotélicienne s'identifie, ou est assimilable, à la lumière naturelle pascalienne - auquel cas les principes auraient chez nos deux auteurs même origine? L'hypothèse est envisageable. La seule différence notable consiste dans le fait que pour Aristote le raisonnement et l'intuition proviennent tous deux de la même faculté, à savoir la raison; Pascal distingue, quant à lui, deux facultés différentes : le cœur, qui saisit les premiers principes, et la raison qui développe les démonstrations ; il note ainsi : « Instinct et raison, marque de deux natures ", dans le fragment 144 des Pensées. La distinction pascalienne possède, en réalité, une raison d'être théologique: si la raison a été entièrement corrompue par le péché originel et que rien "suivant la seule raison n'est juste de soi » (fr. 94), le cœur se pose, en revanche, comme une faculté faillible, certes, mais saine. Il constitue ainsi en nous une réminiscence invincible de l'état pré-adamique dans la mesure où il n'a pas été corrompu comme la raison; il connaît ainsi de façon certaine, mais il ne connait pas tout. J. Laporte affirme :

Le cœur est la faculté de l'infini, il atteint la vérité et la possède mais pour lui seul ${ }^{29}$.

Enfin, Pascal retrouve la logique aristotélicienne sur deux autres points. Tout d'abord, il opère une distinction entre l'évident et le démontrable - distinction que nous ne retrouvons pas du tout aussi clairement formulée chez Descartes. Pour l'auteur des Provinciales, ne pas discerner l'un de l'autre relèverait de la bêtise ou de l'ignorance; distinction qui revient toujours à privilégier, d'un point de vue logique et axiologique, l'évident sur le démontrable. En effet, en soulignant dans son opuscule De l'esprit géométrique, que les choses les plus simples ne peuvent être définies en raison de leur extrême simplicité ${ }^{30}$, Pascal réaffirme, fût-ce de façon fortuite, l'adage aristotélicien: l'évidence ne se démontre pas. Cette absence de démonstration est signe, pour Aristote comme pour Pascal, de sa supériorité. Tout discours est un détour dont peut fort bien se dispenser l'évidence, laquelle engendre une certitude immédiate. Cette évidence, voulant la clarifier, nous ne ferions que l'obscurcir. 

second point est l'acception de la notion de vraisemblable en logique, défini comme accord sur des principes communs. Dans la seconde section de son opuscule, Pascal écrit :

[les axiomes] dès qu'ils sont admis, sont aussi puissants, quoique faux, pour emporter la créance, que les plus véritables ${ }^{31}$.

La qualité première et décisive de l'axiome n'est donc pas sa fausseté ou sa vérité, mais son admission formelle, pourrions-nous dire, laquelle s'appuie sur une puissance très souvent analysée par Pascal : celle de l'habitude. Un axiome admis par plusieurs a valeur d'autorité; ainsi, le meilleur critère de vérité est d'appartenir à la coutume. Or, nous retrouvons ici une considération qui ouvre les Topiques: Aristote y envisage, comme

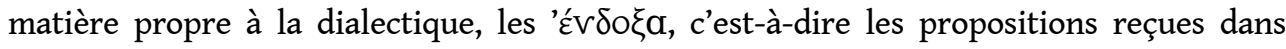
l'opinion. Cette opinion est également source d'autorité pour le Stagirite dans la mesure où elle émane du vulgaire et se trouve renforcée par l'habitude. Une telle affirmation constitue une grande nouveauté par rapport à Platon qui, lui, distinguait soigneusement, à la suite de Parménide, l'opinion de la science et n'accordait aucune attention à la notion de vraisemblable ou encore d'«opinion vraie». Nous pouvons noter que le Théétète, dialogue dans lequel Platon se demande si l'opinion vraie peut s'enseigner, est un dialogue aporétique. Aristote pense, lui, que la vérité de la science est déjà contenue dans certaines opinions à l'état enveloppé. Pascal, dès lors qu'il privilégie comme Aristote l'opinion commune, adopte la même méthode : celle de l'immanence. Il s'agit, en effet, pour l'un comme pour l'autre, de partir d'opinions opposées, puis d'opérer le tri nécessaire afin de discerner, parmi elles, la vérité. Pour Aristote, ces opinions reçues peuvent être vraies ou fausses, et en cela sont proches des axiomes pascaliens ; celles-ci sont cependant plus fiables dans la mesure où, d'une manière plus ou moins directe, elles mènent aux principes :

Le cheminement nécessaire est donc de parvenir à chacun de ces principes en partant des opinions reçues à partir de chacun d'eux ${ }^{32}$.

Le postulat sur lequel semble reposer tout ceci est qu'il y a, entre l'esprit humain et le réel, une entente préalable, une connivence naturelle telle que le fonctionnement naturel de l'esprit le met infailliblement en possession de quelques vérités. Cette dernière assertion est, elle, contraire à la pensée de Pascal, pour lequel l'esprit humain, du moins dans un premier temps, ne se meut que dans l'erreur; et s'il y a connivence préalable, ce serait plutôt entre la raison et l'erreur qu'entre la raison et la vérité. Ainsi, alors qu'Aristote dans les premières lignes de la Métaphysique, souligne l'attrait qu'exerce le vrai sur l'homme en énonçant " Tout homme désire naturellement savoir... », et peut bien atteindre le vrai, Pascal insiste sur l'incapacité de l'homme à connaitre le vrai :

L'homme n'est qu'un sujet plein d'erreur naturelle... [Rien] ne lui montre la vérité.

Tout l'abuse. (fr. 78)

Telle est la triste condition de l'homme sans Dieu. Au principe dogmatique d'intelligibilité universelle, Pascal substitue son principe d'intelligibilité limitée. Le postulat aristotélicien ne saurait donc être admis par Pascal ; pour le premier, la vérité l'emporte sur le mensonge; pour le second, c'est l'inverse. C'est d'ailleurs pourquoi, dans les principes de l'art de persuader, l'opinion du vrai se substitue, pour Pascal, au vrai.

Cependant, nos deux auteurs envisagent à partir d'un point de départ commun, les possibilités de convaincre, en analysant des schémas formels de situation: dans l'Art de persuader, Pascal étudie la "qualité des choses que nous devons étudier » et distingue trois cas types : celui où «les [choses que nous voulons persuader] se tirent, par une conséquence nécessaire, des principes communs et des vérités avouées; [celui où] elles 
ont une union étroite avec les objets de notre satisfaction ; [celui où elles sont] contraires aux plaisirs qui nous touchent le plus ${ }^{33}$ ». D'une manière analogue, Aristote, dans les livres VI et VIII des Topiques, opère lui aussi une réduction des types de problème à poser et n'en conserve que quatre : suivant que l'attribut appartient au sujet dont il est question (livres II et III), comme un de ses accidents ou un de ses propres (livre V), ou comme son genre (livres VI et VII). Puis pour chacun de ces chefs d'attribution, il énumère les lieux, c'est-à-dire les sources d'argumentation, pour ou contre l'attribution de tel attribut à tel sujet et à tel titre. Le procédé est analytique, car dans la persuasion, l'infaillible est le déductif et il faut, dès lors, remonter sans cesse aux principes. Pascal note à ce propos, dans l'Art de persuader :

[que] en montrant le rapport qu'ont [les choses que l'on veut persuader] avec les principes accordés, il y a nécessité inévitable de convaincre ${ }^{34}$.

Cependant, alors qu'Aristote part des opinions répandues en général, Pascal considère celles de son interlocuteur : il y a donc une différence de point de vue notoire. Aristote aborde le problème de manière beaucoup plus abstraite, sinon théorique : il considère le point de vue exclusif de la raison et procède ainsi à l'examen de toutes les opinions concevables, même si certaines n'ont été soutenues par personne. Pascal, lui, considère cet art de persuader et dans son rapport à l'intelligence - ce qui donne lieu à une multiplicité d'opinions possibles -, et dans son rapport à la volonté - ce qui donne lieu à une multiplicité de dispositions possibles : en cela, il élabore une rhétorique qui dépasse celle d'Aristote, car elle prend en compte l'ensemble de la psychologie humaine. S'il y a un identique effort pour répertorier les différents cas de figures, Aristote envisage des cas théoriques, des lieux de persuasion; Pascal, en revanche, étudie les différentes situations dans lesquelles un individu concret - en l'occurrence son interlocuteur - peut se trouver. Ainsi les premières lignes de l'Art de persuader mettent en relief l'aspect novateur de la rhétorique pascalienne :

L'art de persuader a un rapport nécessaire à la manière dont les hommes

consentent à ce qu'on leur propose ${ }^{35}$,

rapport qui précisément échappe à l'analyse aristotélicienne. Pour Pascal, seul peut être convaincu celui qui y est effectivement disposé : dès lors, tout art de persuader se décompose en art de convaincre qui s'adresse à l'entendement, à l'instar des Topiques, et en art d'agréer, qui s'adresse à la volonté. Si le premier vient prendre appui sur des fondements aristotéliciens, le second ne s'y réfère pas : en effet, il constitue un apport spécifiquement pascalien à la rhétorique classique.

\section{II. - La physique}

31 Du point de vue des mathématiques, de la géométrie et de la physique, la rupture marquée par Pascal avec la pensée aristotélicienne est sans nul doute plus patente parce qu'elle vient s'inscrire dans une période de complète mutation. En effet, l'ensemble du $\mathrm{XVII}^{\mathrm{e}}$ siècle, du point de vue mathématique et physique - avec un Galilée et un Descartes - marque une rupture par rapport aux connaissances antiques et médiévales. A. Koyré a fort bien décrit, dans son ouvrage Du monde clos à l'univers infini, la révolution qui s'opère entre la destruction du cosmos aristotélicien, dans lequel la structure spatiale incarnait une hiérarchie de valeurs, et l'apparition d'un nouveau concept, celui d'espace qui se trouve être un espace géométrique ${ }^{36}$. Et c'est bien dans un tel contexte qu'il nous faut replacer notre étude comparative. 

véritable méthode scientifique, présentée sous la forme d'une axiomatique, c'est-à-dire sous une forme strictement déductive. À l'instar de Descartes, dans les règles de la méthode, Pascal s'attache donc à suivre l'ordre des raisons et, en cela, s'éloigne radicalement de la voie naturelle d'Aristote. Il note ainsi que toute affirmation doit être ou bien un principe - qui est de lui-même « clair et distinct aux sens et à la raison »-, ou bien se déduire par "des conséquences infaillibles et nécessaires ${ }^{38}$ » de tel principe. D'autre part, cette méthode est très largement une méthode expérimentale: en effet, dans cette même lettre, Pascal analyse de manière schématique les rapports de l'hypothèse à l'expérience et détermine ainsi un mouvement en trois temps: poser l'hypothèse, l'éprouver par l'expérience et la qualifier de manière certaine en fonction de cette expérience. Car c'est bien cette dernière qui a le dernier mot. Pascal n'hésite pas à parler de l'évidence de l'expérience. Il note ainsi dans l'avis au lecteur de son Récit de la grande expérience de l'équilibre des liqueurs :

l'évidence des expériences me force de quitter les opinions où le respect de l'antiquité m'avait retenu ${ }^{39}$.

L'expérience fonctionne donc comme un critère à la fois sûr et simple, qui n'offre pas de complexité à l'analyse mais oblige pour ainsi dire à la réflexion. Pascal affirme au chapitre VII de son Traité de l'équilibre des liqueurs que

il ne faut pas avoir beaucoup de lumière pour tirer de cette expérience tout ce que nous avions déjà démontrée ${ }^{40}$.

La pensée va donc de la nature à la raison, mouvement propre à la démarche du physicien moderne. L'expérience offre bien les deux caractéristiques fondamentales de l'évidence - et Pascal est ici plus proche de Descartes que d'Aristote -, qui sont la clarté et la distinction. Dans la physique, les expériences ont bien plus de force pour persuader que les raisonnements. Nominaliste par ses exigences de physicien, Pascal refuse cependant au concept toute valeur normative, tout contenu notionnel: son véritable maître est la seule expérience ${ }^{41}$. Aussi soumet-il tantôt l'idée de nature à l'expérience pour en faire apparaitre l'irréalité (idée dotée chez Aristote d'une véritable valeur normative), tantôt l'expérience à l'idée de nature pour faire éclater l'absurdité de celle-ci. Il opère ainsi une 
critique du réel par la pensée et une critique de la pensée par le réel, balancement propre au nouvel esprit scientifique.

Or, nous retrouvons exprimée ici la question débattue par Pascal dans sa correspondance à propos des expériences sur le vide, notamment dans une lettre adressée au Père Noël ${ }^{42}$ et une autre à Le Pailleur. L'auteur des Provinciales y opère une critique vigoureuse de certains concepts aristotéliciens à l'occasion de la querelle sur l'existence du vide. Aristote, en effet, a nié son existence au livre IV de sa Physique, négation qui met fin à une double réflexion sur la nature du lieu et sur celle du vide, la seconde étant inséparable de la première. Partant de la nécessité du mouvement pour toute recherche sur le lieu, et de la distinction du mû par soi et du mû par accident, il montre, en un premier temps, que le lieu est essentiellement séparable; dès lors, procédant par élimination successive de toutes les hypothèses possibles, il affirme que le lieu ne peut être ni forme, ni matière, ni intervalle mais seulement la limite immobile de l'enveloppe. Comme selon la définition populaire (bel exemple d"ع́ $\vee \delta \circ \xi o v$ ), le vide est, lui, un lieu privé de corps, il convient d'étudier, après le lieu, le vide. Or, l'argument principal des partisans de l'existence du vide consiste à affirmer qu'il ne saurait y avoir de mouvement local sans vide; à quoi Aristote répond que, d'une part, le mouvement local des choses est un mouvement naturel qui ne requiert donc pas de cause externe; et que d'autre part, la vitesse d'un corps est précisément déterminée par sa résistance au milieu. Dans le vide, la vitesse serait infinie. Le vide ne peut donc pas être un lieu où il n'y a rien, puisque, d'après sa définition ${ }^{43}$, il est la limite immobile de l'enveloppe, c'est-à-dire que le lieu est à la chose ce que la limite est au limité : il ne peut donc exister de lieu sans chose.

Enfin, en considérant le vide en lui-même et pas seulement dans les conséquences qu'il permet, on s'aperçoit qu'à côté de l'extension pure d'un corps, il ne faut pas poser un vide sous peine de poser un vide inutile, doublure de la chose. De même, la rareté et la densité ne s'expliquent pas par une introduction ou une élimination du vide -comme l'affirmaient ses partisans -, mais la difficulté réelle de l'interaction des parties de l'univers peut être parfaitement résolue par la théorie de la matière, sujet unique des deux contraires et contenant l'un des deux en puissance. L'argumentation d'Aristote est de fait assez serrée car les concepts de mouvement, de lieu et de corps s'imbriquent étroitement. Mais de cette rigoureuse explication, un seul principe avait été retenu, parvenu jusqu'à Pascal sous la forme d'une maxime : « La Nature a horreur du vide »

Pascal, par la fameuse expérience du puy de Dôme, du 19 septembre 1648, nous fournit un résultat inverse à cette théorie. Pour ce faire, il utilise un tube rempli de vif-argent, qu'il transporte à diverses hauteurs; or, la hauteur du vif-argent est moindre au haut qu'au bas de la montagne, ce qui prouve que la cause de cette suspension est non l'horreur du vide, mais bien la pesanteur et la pression de l'air. Cette simple expérience qui va à l'encontre d'opinions répandues depuis l'Antiquité, conduit Pascal à une remise en question, du point de vue théorique, de notions aristotéliciennes. Au concept unique de lieu, Pascal substitue deux notions : celle d'espace :

dont l'amplitude embrasse tout, qui est aussi stable et immobile en chacune de ses parties comme il l'est aussi en son total ${ }^{44}$,

et celle de place, qui est «immobile, ferme et inébranlable ${ }^{45}$ ». Il montre ainsi que le Père Noël suppose des relations analytiques entre les termes - solide, lumière, mouvement qui ne sont en fait dues qu'à des circonstances expérimentales particulières. On assiste ainsi à la substitution d'un univers défini comme un ensemble différencié de lieux, élaboré dans Physique IV, par un nouvel espace, celui de la géométrie euclidienne, dont 
l'extension homogène est infinie. Il est à noter que Pascal aboutit à la même conclusion qu'Aristote : cet espace n'est « ni corps, ni esprit, ni accident, ni Dieu, ni créature ${ }^{46} »$. Mais il va plus loin que le Stagirite et formule le concept moderne d'espace. Ainsi note-t-il dans le principe $\mathrm{n}^{\circ} 7$ de son Introduction à la géométrie que «les points ne diffèrent que de situation ». C'est ce que ne peut comprendre le Père Noël, pour qui «tout ce qui est espace est corps ${ }^{47}$ ». Ce principe est en parfaite opposition avec la théorie aristotélicienne des lieux : Aristote distinguait, quant à lui, du fait de l'immobilité du cosmos, une gauche et une droite absolues.

Les espèces et différences du lieu sont haut, bas, avant, arrière, droite, gauche et ces

distinctions valent non pas relativement à nous, mais dans le tout lui-même ${ }^{48}$.

La stabilité de son univers empêche Aristote de percevoir la véracité de la théorie de la relativité. La définition pascalienne de l'espace permet, au contraire, son élaboration. Ainsi que le dénonce Pascal dans sa lettre à Le Pailleur, le Père Noël élabore un nouveau concept de corps, étranger à Aristote, quand il écrit que :

[le corps est] ce qui est composé de parties les unes hors les autres, (...) que tout corps est espace quand on considère entre les extrémités, et que tout espace est corps, puisque tout espace est composé de parties les unes hors les autres ${ }^{49}$.

41 Il utilise ainsi un même terme pour plusieurs définitions - le corps est également un composé de matière et de forme -, et fait là œuvre de sophiste. Un point nous aidera sans doute à mieux comprendre le différend qui oppose Pascal au Père Noël. L'auteur des Pensées, en effet, a suivi Descartes dans son identification de la matière et de l'espace - l'un n'existe qu'en fonction de l'autre, et, par conséquent, a fortement réduit le concept aristotélicien de matière, lequel désignait à la fois la matière comme principe d'individuation et la matière comme pure puissance. D'autre part, le concept de mouvement, dans la philosophie aristotélicienne, avait une acception beaucoup plus large et désignait, outre le mouvement physique, le passage de la puissance à l'acte ; ce dernier sens, en effet, est exclu de l'univers pascalien. Or là réside sans doute l'une des différences fondamentales entre la physique aristotélicienne et la physique moderne qu'élabore Pascal : la première est à ce point fondée sur une métaphysique qu'il devient difficile de les distinguer - ainsi en est-il du concept de mouvement, concept tant physique que métaphysique; la seconde s'élabore parfois en marge de la métaphysique traditionnelle. De plus, chez Pascal tout particulièrement, une telle interaction entre ces deux sciences n'existe pas.

C'est pour cette même raison que Pascal et Aristote ne peuvent pas non plus s'accorder sur le concept d'infini. Aristote utilise ce concept en parfait physicien. Dans le chapitre IV de la Physique, le Stagirite s'intéresse à l'infini comme concept voisin de la limite et donc du lieu. Il énumère, tout d'abord, les raisons que nous avons de croire à l'infini, raisons qu'il chiffre au nombre de cinq: l'infini du temps, la divisibilité des grandeurs, le caractère incessant de la génération, la notion de limite - dans la mesure où le limité étant limité à autre chose, rien ne sera limité s'il faut que la limitation se fasse entre deux termes -, et enfin l'accroissement sans fin des grandeurs numériques ou de la progression vers un espace extérieur au monde. Mais, souligne Aristote, si cet accroissement nous paraît sans fin, cela est surtout dû à l'impuissance de notre faculté de représentation. Notons que sur ce point précis du rôle de l'imagination, Pascal est en parfait accord avec ce dernier lorsqu'il écrit :

Nous avons beau enfler nos conceptions au-delà des espaces imaginables, nous

n'enfantons que des atomes au prix de la réalité des choses. (fr. 230) 
Cette faiblesse de l'imagination conduit Aristote à affirmer qu'il serait absurde de se fonder sur la représentation, car l'excès et le défaut ne se produisent pas dans la chose, mais dans la représentation ${ }^{50}$; elle trouve ainsi un écho dans la condamnation pascalienne de cette même faculté.

Le Stagirite entreprend ensuite de déterminer la nature de cet infini et distingue alors l'infini réel, de l'infini mathématique. Le premier, en effet, ne saurait exister car il serait en parfaite contradiction avec la théorie aristotélicienne des lieux qui veut que tout corps se trouve dans un lieu naturel déterminé et fini : l'infini ne pourrait ainsi être situé ; quant aux mathématiciens, Aristote affirme qu'ils n'ont pas besoin de cette notion d'infini mais seulement de celle de grandeur, aussi grande que possible mais limitée. Cependant, il reconnaît qu'il serait incohérent de nier totalement l'infini : on ne saurait s'en passer en considérant l'infinité des nombres. Aussi lui attribue-t-il une certaine existence mais d'un ordre inférieur : celle de la puissance. Ainsi, si la grandeur n'est pas infinie en acte, elle l'est cependant par division, c'est-à-dire potentiellement. L'infini n'existe donc que par réduction ou par composition. Cette dernière assertion conduit le Stagirite à formuler une nouvelle définition de l'infini :

L'infini se trouve donc être le contraire de ce qu'on dit: en effet, non pas ce en dehors de quoi il n'y a rien, mais ce en dehors de quoi il y a toujours quelque chose $\mathrm{e}^{51}$

définition qui souligne l'opinion dépréciative que le Stagirite se fait de la notion d'infini. Aristote lui confère, en effet, l'acception opposée à celle des termes «achevé, entier, parfait ». Car l'infini est ce au-delà de quoi il y a toujours quelque chose de nouveau ; inversement, ce qui est achevé est ce d'où rien n'est absent. Mais rien n'est achevé s'il n'est terminé, or le terme est la limite; l'infini est donc, pour Aristote, l'archétype de l'imparfait.

analyse, quant à lui, l'infini de deux manières : d'une part, dans le Traité de la sommation des puissances numériques, il l'étudie dans son rapport à la divisibilité des nombres; de ce point de vue, il parvient aux mêmes conclusions qu'Aristote ; d'autre part, dans son apologie, il le mentionne à plusieurs reprises pour désigner à la fois l'infini de l'univers visible et l'infini divin. Si nous prenons ce texte canonique qu'est le fragment sur les deux infinis, nous y découvrons tout à la fois la méthode et le but de l'analyse pascalienne. Le but nous est livré par le titre même du fragment : il s'agit de montrer la disproportion de l'homme par rapport à l'infiniment grand et par rapport à l'infiniment petit. L'espace pour Pascal ne connaît pas un repos absolu mais une perpétuelle expansion ; contrairement à l'univers hiérarchiquement ordonné de haut en bas et de gauche à droite d'Aristote, dans lequel le sens a toujours une signification absolue, l'univers décrit par Pascal est construit en abîmes, ce qui produit un effet de vertige, synthétisé dans l'affirmation suivante :

C'est une sphère infinie dont le centre est partout, la circonférence nulle part. (fr. 230)

La contemplation de l'univers quasi immobile d'Aristote est une contemplation sereine, voire sécurisante; celle de l'univers pascalien déstabilise ${ }^{52}$. Pascal nous le précise luimême :

Ne cherchons donc point d'assurance et de fermeté (...) Rien ne peut fixer le fini entre les deux infinis qui l'enferment et le fuient. (fr. 230) Or, c'est précisément dans ce rapport du fini à l'infini que Pascal diffère radicalement d'Aristote : pour ce dernier, l'infini, l'inachevé était synonyme d'imparfait, alors que le 
fini, existant en acte, atteignait sa plénitude; pour Pascal, en revanche, l'infini est la marque du divin ${ }^{53}$. En effet, le seul véritable infini est Dieu, être incréé ; mais toute chose créée - «la nature ayant gravé son image et celle de son auteur dans toutes choses » (fr. 230) - tient de cette double infinité. Dans le fragment 680 des Pensées, Pascal établit une progression du fini à l'infini visible, puis à l'infini invisible : nous connaissons la nature du fini, parce que nous sommes finis et étendus comme lui, nous connaissons l'existence de l'infini visible, parce qu'il a une étendue comme nous, mais nous ignorons sa nature ; enfin, nous ne connaissons ni l'existence ni la nature de l'infini invisible, parce qu'il n'a ni étendue ni borne. Or, cette hiérarchie fonctionne également comme une hiérarchie de valeurs ${ }^{54}$ : l'infini relève de Dieu et le fini du néant : le rapport est inversé : «Le fini s'anéantit en présence de l'infini» (fr. 680). En réalité, le point précis de désaccord entre Pascal et Aristote est la notion même de puissance: en effet, alors qu'Aristote l'emploie pour désigner l'infini inachevé, Pascal l'emploie pour désigner Dieu, plénitude de l'être. Pour Aristote, l'acte pur exclut l'infini qui est illimité, et par là même pure possibilité : la puissance active est inséparable pour ce dernier de la puissance passive et elle se trouve donc étrangère à Dieu. Pascal, en revanche, reprend la distinction thomiste de puissance passive qui désigne la matière, et de la puissance active qui désigne une activité parfaite, agissant selon la libre détermination d'une volonté qui se possède et qui n'est déterminée par rien. Le fossé qui sépare ici nos deux auteurs est celui qui sépare la philosophie grecque de la philosophie chrétienne: de la notion moderne d'infini, - entendu comme parfait - et de celle, corrélative, de puissance infinie au service d'une volonté infinie, résulte l'intelligibilité de la création, concept entièrement étranger à la philosophie préchrétienne. Le Dieu de Pascal vit, agit et, par là même, est à l'origine de tout être ; celui d'Aristote, n'est que « pensée de sa pensée $e^{55}$ ».

En revanche, il est un point sur lequel nos deux auteurs s'accordent : en effet, ni pour l'un ni pour l'autre, leur physique n'aboutit à la mise en place du concept de création. C'est principalement dans la Physique et dans la Métaphysique, qu'Aristote nous fournit son explication de l'origine et du fonctionnement de l'univers. Dans la Physique, Aristote démontre tout d'abord la perpétuité du mouvement : en effet, comme il ne peut y avoir rien d'antérieur et de postérieur sans le temps, il ne peut y avoir quelque chose d'antérieur au temps; le temps n'a donc pas commencé et le mouvement non plus, puisque le temps est la mesure du mouvement. Il en cherche ensuite la cause : si tout ce qui est mû est mû par autre chose et par une chose mue, il faut qu'il y ait un premier moteur qui ne soit pas mû par quelqu'autre chose ; de plus, si le premier moteur, tout en étant mû, ne peut pas l'être par autre chose, il est nécessaire qu'il soit mû par soi. Mais dans ce qui se meut par soi-même, il y a une partie qui meut et une partie qui est mue, et cette partie qui meut est motrice mais immobile, sinon par quoi serait-elle mue ? Et si elle était mue, pourrait-on dire encore que ce moteur est premier?

On voit donc d'après ce qui précède que le premier moteur est immobile ${ }^{56}$.

Et puisque le mouvement est éternel, ce premier moteur est éternel et immobile. Enfin, dernière caractéristique, ce premier moteur est impassible: alors que tout moteur qui meut, agit par contact et subit donc lui-même une réaction, le premier moteur lui " touche le mobile tout en étant lui-même touché par rien ${ }^{57}$." Cette conception du premier moteur va de pair avec celle du mouvement, défini comme passage de la puissance à l'acte, et il faudra attendre le $\mathrm{XVII}^{\mathrm{e}}$ siècle pour qu'apparaisse une mathématisation de la physique qui mette au premier plan la notion de mesure, et écarte celle de puissance et d'acte. La notion de création est ainsi résolument étrangère à la 
philosophie aristotélicienne. Pascal, quant à lui, oblitère volontairement, dans le fragment 230 , ce point de jonction entre physique et métaphysique. Oscillant entre une dénégation de toute théologie naturelle et une affirmation simultanée de son existence et de sa vanité, l'auteur des Pensées se refuse à tirer une conclusion. Ce concept qui marque traditionnellement une rupture entre la pensée grecque et la pensée chrétienne n'opère ici aucun clivage ${ }^{58}$.

\section{III. - La métaphysique}

51 Une étude rapide de la cosmologie aristotélicienne nous permet de distinguer les principaux concepts métaphysiques qui la sous-tendent, notamment ceux de nature, de substance et de vérité, autant de concepts qui permettent d'unifier des réalités complexes : celle du corps et de l'âme, de l'un et du multiple, de l'idée et de la chose, et d'organiser des êtres différents. Dans quelle mesure ces concepts sont-ils remis en cause par Pascal? Le premier point de la métaphysique aristotélicienne que nous allons considérer, est celui de la hiérarchie des êtres.

Dès le début des Parties des Animaux, Aristote explique qu'il distingue l'âme végétale de l'âme animale, et celle-ci de l'âme humaine qui, de nature proprement spirituelle, est immortelle. L'homme se trouve donc au sommet de la hiérarchie. Par son intelligence, il touche presque au divin. Ainsi s'établit la hiérarchie des êtres en rapport avec les fonctions de l'âme, et ce, de manière régulière. Pascal, comme Aristote, adhère à une vision du monde où le spirituel l'emporte sur le matériel. On retrouve un écho de la hiérarchie aristotélicienne dans le fragment 143: «Mais je ne puis concevoir l'homme sans pensée. Ce serait une pierre ou une brute ", et dans les fragments qui traitent de la différence et de la ressemblance entre l'homme et l'animal ${ }^{59}$. Mais il est sans doute plus intéressant d'associer à cette vision hiérarchique de l'univers, la conception des trois ordres que Pascal développe au fragment 339 des Pensées:

La distance infinie des corps aux esprits figure la distance infiniment plus infinie des esprits à la charité, car elle est surnaturelle.

Il s'agit bien également d'un ordre hiérarchique, mais celui-ci connaît, à chaque degré, une différence "infinie ", une véritable solution de continuité. Pascal écrit: "Ce sont trois ordres différents. De genre» (fr. 339). L'une et l'autre hiérarchie possèdent une même caractéristique : chaque genre y implique ceux qui le précèdent, sans impliquer forcément ceux qui le suivent. Certes, Pascal et Aristote ne travaillent pas sur la même matière: l'un étudie les fonctions de l'âme, l'autre les différentes sphères - pour employer un langage kierkegaardien - où se meut un chrétien. Cependant, il y a une similitude de vision d'ensemble et cela, sans doute, parce que nos deux auteurs, cherchant à situer l'homme par rapport à Dieu, proposent une vision ascendante de l'homme vers Dieu et non circulaire, où l'homme serait pris comme centre. La différence d'ordre est, pour Pascal, une différence de nature et c'est pourquoi le fossé ne saurait être comblé : ainsi en est-il du matériel (ordre de la chair), et du spirituel (ordre de l'esprit). De ce point de vue, la hiérarchie pascalienne est bien alors superposable à celle d'Aristote, qui considère les différentes natures de l'âme. Cependant, la théorie des trois ordres de Pascal connaît un écart flagrant qui n'apparaît pas chez Aristote : c'est l'écart qui sépare l'ordre de l'esprit de l'ordre de la charité. En effet, comme le souligne J. Mesnard, nous pouvons toujours réduire les structures ternaires de Pascal à deux éléments : la chair et l'esprit peuvent être ainsi momentanément réunis pour s'opposer à la charité ; ainsi 
demeure le naturel (chair et esprit) qui s'oppose au surnaturel (la charité60). Aristote, lui, ne propose de vision hiérarchique que de la seule nature. Il ignore donc le troisième ordre mais partage, avec l'auteur des Pensées, une vision du monde organisée et surtout finalisée.

Aussi n'est-ce pas un hasard si une expression aussi typiquement aristotélicienne que celle du «propre de » apparaît sous la plume de Pascal, expression qui n'a de sens que dans un monde parfaitement structuré. Aristote l'emploie lorsqu'il cherche à définir le bonheur. Il tente alors de découvrir la fonction propre de l'homme :

Or, ce que nous cherchons, c'est ce qui est propre à l'homme ${ }^{61}$.

Nous avons déjà considéré la structure métaphysique de l'être chez Aristote et distingué la matière de la forme : le propre d'un genre est précisément lié à cette dernière, qui est indifféremment forme, fonction ou fin. Cette expression «le propre de » renvoie donc toujours à un monde d'êtres finalisés; Pascal, comme Aristote, cherche la fin ultime de l'homme. Mais elle fait également référence à une vision statique de l'univers où chaque élément possède une place déterminée dont il ne saurait se mouvoir. Nous entrevoyons ici la richesse d'une telle expression qui nous livre une conception physique, métaphysique et morale du monde. L'excellence y est précisément de savoir demeurer à sa place et de maintenir ainsi la stabilité de l'ensemble. Pascal utilise, lui aussi, cette expression en ce sens : «Le propre de chaque chose doit être recherché » (fr. 650). Le fragment qui précède nous permet, en effet, de mesurer l'enjeu qu'elle contient :

Dieu a tout créé pour soi, a donné puissance de peines et de biens pour soi. Vous pouvez l'appliquer à Dieu ou à vous. Si à Dieu, l'Évangile est la règle. Si à vous, vous tiendrez la place de Dieu. (fr. 649)

L'homme est ainsi, à la suite du péché originel, placé devant l'alternative suivante: rechercher ce qui lui est propre, c'est-à-dire la fin que Dieu lui a assignée et l'accomplir selon la règle que Dieu a donnée, ou rechercher ce qui est le propre de Dieu et désirer alors prendre sa place. L'affirmation de Pascal, qui relève tant de la métaphysique que de l'eschatologie, prend dès lors un relief nouveau: il s'agit, en effet, de chercher à maintenir l'équilibre voulu par Dieu et de respecter ainsi son ordonnance. L'expression «le propre de» ne fait ainsi pas tant référence à un monde statique qu'à un monde parfaitement ordonné, dont le mouvement est prévu parce que voulu par Dieu.

D'autres notions de la métaphysique aristotélicienne subissent, en revanche, une modification notoire : la première est celle de substance, qui perd, dans les Pensées, toute force unificatrice pour ne devenir qu'un mot sans contenu ${ }^{62}$. Là où Aristote montrait l'unité, Pascal montre la scission. Dans le Traité de l'Âme et dans la Métaphysique, le Stagirite résout la question de l'union de l'âme et du corps, en affirmant que l'homme est un être composé et que cette composition n'est pas une simple addition, mais quelque chose de dynamique. En effet, toute substance est composée de matière et de forme : la matière désignant une pure potentialité, elle doit être informée par une forme substantielle. Ce qui est cause de l'être n'est ni la forme, ni la matière mais bien l'union des deux. Aristote écrit au livre $\mathrm{Z}$ de la Métaphysique :

Ce qui est composé de quelque chose de telle sorte que ce tout soit un, est semblable non à une pure juxtaposition mais à la syllabe : or, la syllabe n'est pas ses lettres composantes : BA n'est pas identique à $\mathrm{B}$ et $\mathrm{A}$.

On peut penser qu'il existe un principe d'union, qui tout en n'étant pas un élément est cause du moins que ceci est de la chair, et cela une syllabe; et de même pour tous les autres composés. Or, ce principe d'union c'est la substance de chaque chose car c'est là la cause primordiale de son être (...). La substance est cette sorte de 
nature qui est non pas un élément matériel mais un principe formel, l'élément étant ce en quoi la chose se divise et qui est présent en elle comme matière : par exemple, pour la syllabe, les lettres A et $\mathrm{B}^{63}$.

Elle comprend, en outre, un principe d'union qui n'est pas lui-même un élément ; il en est de même pour l'homme, composé de matière et de forme, et d'un principe d'union qui est précisément la substance. Or, cette unité de l'être éclate chez Descartes et plus encore chez Pascal. Séparant l'âme du corps, l'esprit de la matière, ce dernier ne trouve pas d'explication rationnelle à leur union :

L'homme ne peut concevoir ce que c'est que corps, et encore moins ce que c'est qu'esprit, et moins qu'aucune chose comment un corps peut être uni avec un esprit. C'est là le comble de ses difficultés, et cependant c'est son propre être. (fr. 230)

Comme nous l'avions déjà noté à propos de la physique, les concepts clefs de l'aristotélisme (matière et forme, puissance et acte) qui permettaient d'unifier la réalité, ne sont pas connus de Pascal. Cette scission au sein de la nature humaine semble parfois sans appel : «Instinct et raison, marque de deux natures » (fr. 144); et la matière, séparée de l'âme, principe de vie, doit trouver en elle-même la cause de son dynamisme. Le principe de mouvement de la matière doit être la matière elle-même. Ainsi, en refusant le finalisme qu'engendrait la notion de forme, Pascal insiste sur l'automatisme de l'instinct et accepte « en gros » le mécanisme cartésien :

Descartes. Il faut dire en gros : « Cela se fait par figure et mouvement », car cela est

vrai. Mais de dire quelles et composer la machine, cela est ridicule (fr. 118),

même si cette explication ne saurait suffire. L'union du corps et de l'âme n'est dès lors plus substantielle mais accidentelle: notre âme est «jetée dans notre corps» (fr. 680). Pascal présente ainsi à notre réflexion un monde et un homme éclatés, auxquels manque cruellement un principe d'unité. Cela relève, ne l'oublions pas, du dessein de Pascal dans la première partie de l'apologie: montrer le désordre et l'absurde du monde et de l'homme, sans Dieu. Aussi le concept de nature subit-il le même sort.

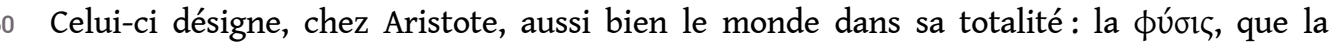
nature propre à chaque être. Cette dernière se trouve définie de la manière suivante :

Un principe et une cause de mouvement et de repos pour la chose en laquelle elle réside immédiatement et non par accident ${ }^{64}$.

Elle est un principe interne et se distingue en cela de la technique, qui est « un principe de mouvement résidant dans une autre, tandis que la nature est un principe résidant dans la chose même ${ }^{65}$ ». La nature, principe de mouvement et de croissance, en produisant le changement, le produit vers une fin, et cette fin n'est autre que la forme vers laquelle et par laquelle se meuvent les êtres naturels. Ainsi, en tant que forme, la nature agit en vue d'une fin qui n'est autre qu'elle-même. Tous les êtres sont donc finalisés et la nature est fin en même temps que norme : le normal est le naturel. Le concept de limite marque la séparation, du point de vue métaphysique, entre le naturel et le dénaturé: nous le retrouverons du point de vue moral. La nature, qui peut être ainsi considérée comme cause finale, fonctionne également comme critère. Ce dernier point, celui d'une nature/ référence, est sérieusement battu en brèche dans les Pensées. Si le concept renvoie également, pour Pascal, à celui de mouvement et de fin, celui d'une totalité signifiante, d'une réalité en soi qui promet une intelligibilité totale de l'être, résume pour lui « toutes les fascinations de la métaphysique en l'exigence qu'elle porte d'une rationalité intégrale ${ }^{66}$ ». Aussi cherche-t-il constamment à faire apparaître une distance entre la nature effective et l'idée de nature, et à repousser la tentation métaphysique de prendre ses

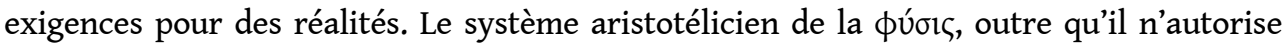


qu'un déisme inutile pour le salut, apparaît à Pascal fort contestable, dans la mesure où il se présente comme une rationalisation abusive du réel, imposant à la pensée une logique impropre à régler le donné de l'expérience. Invoquer ainsi une nature, c'est abusivement ériger le fait en droit. Aussi refuse-t-il tout principe a priori dicté par des présupposés métaphysiques, qui ne sont peut-être qu'une ruse de langage, consistant à regrouper sous un seul terme une réalité trop diverse.

L'auteur des Pensées, en revanche, se montre soucieux de respecter la nature dans ses différentes manifestations; notre raison doit toujours se soumettre aux faits, car elle s'alimente ainsi aux sources mêmes du vrai. Or, les faits ne fournissent pas d'emblée leur signification; étant par essence incomplets et transitoires, ils posent davantage de problèmes qu'ils ne résolvent de questions. La réalité est trop complexe pour être, ainsi, analysée et perçue en deux ou trois définitions. L'une de ses premières caractéristiques est sa contradiction interne : le principe d'identité ne conduit à aucune issue : «Chaque chose est ici vraie en partie, fausse en partie » (fr. 450) Cette contradiction vient de notre ignorance de la loi et découle de la déraison du monde, selon la belle expression de L. Thirouin ${ }^{67}$ :

Toutes choses sont sorties du néant et portées jusqu'à l'infini. Qui suivra ces étonnantes démarches? L'auteur de ces merveilles les comprend. Tout autre ne le peut faire. (fr. 230)

Aussi la méthode d'appréhension du réel diffère-t-elle chez nos deux auteurs. L'intelligibilité quasi totale de l'être incite Aristote à prendre pour fil directeur de toute analyse et de toute réflexion le principe de causalité : il lui faut partir des effets pour remonter à la cause et ceci, dans un mouvement simple et unilatéral de la raison. Les faits, dans la mesure où ils sont, sont toujours compréhensibles puisque c'est l'être qui leur confère leur intelligibilité. Les seules conditions à respecter pour qu'ils deviennent objet de science sont le nécessaire et le général.

Pascal, face à l'être dialectique et contradictoire de la nature, ne peut utiliser ce simple principe de causalité : il travaille donc toujours à chercher la " raison des effets ». Cette formulation, quelque peu déroutante, montre qu'il s'agit bien d'un mouvement identique (remonter des effets à la raison des effets), conforme cependant à la vision pascalienne de la réalité : la matière fournie étant non l'intelligible mais l'absurde, le métaphysicien doit savoir discerner les effets à considérer de ceux à négliger. Il ne s'agit plus d'additionner des faits, simples en eux-mêmes, pour en faire ressortir une loi générale, mais plutôt d'en sélectionner certains pour tenter de découvrir la loi qui les régit :

L'effet pascalien n'est pas un phénomène quelconque mais le phénomène choisi pour illustrer une $\operatorname{loi}^{68}$.

Il est le résultat d'une véritable construction intellectuelle. Rendre raison des effets signifie donc reconnaitre et interpréter les contradictions naturelles : le métaphysicien n'est pas tant un spectateur de la réalité qu'un traducteur, un déchiffreur de cette même réalité. Ainsi le principe de causalité, omniprésent chez Aristote, est largement conservé par Pascal, mais adapté à sa conception de la nature. Savoir conserver tout en modifiant, tel semble être le véritable coup de génie de Pascal. Un tel principe vient, de plus, ouvrir les portes à la démarche théologique :

Toute remontée des effets aux causes... participe déjà de ce qui est le sens ultime de nos démarches, c'est-à-dire le passage du matériel à l'immatériel, de la nature à la grâce $^{69}$. 
désaccord sur la notion de nature et sur son intelligibilité, Pascal et Aristote se retrouvent en parfaite syntonie pour affirmer l'incompréhensibilité de l'individuel. L'auteur des Pensées aborde cette question de façon marginale dans le fragment 688 :

Où est donc ce moi, s'il n'est ni dans le corps ni dans l'âme ? Et comment aimer le corps ou l'âme sinon pour ses qualités, qui ne sont point ce qui fait le moi, puisqu'elles sont périssables?

Car aimerait-on la substance de l'âme d'une personne abstraitement, et quelques qualités qui y fussent? Cela ne se peut et serait injuste. On n'aime donc jamais personne, mais seulement des qualités. (fr. 567) constitue le moi. Une telle résistance de l'individuel à entrer dans le cadre d'une définition proviendrait-elle d'impératifs aristotéliciens? En effet, pour Aristote il n'y a de science, et partant de démonstration et de définition, que du nécessaire et du général. Cependant, ce qui est inconnaissable pour le Stagirite, n'est pas l'individuel comme tel, mais l'individuel en tant que contingent. Ce qui pourrait être autrement qu'il n'est, n'est objet ni de démonstration ni de définition ; mais Aristote souligne que s'il se trouvait des choses individuelles desquelles toute contingence fût exclue, elles seraient objet de science $^{70}$. Cette méconnaissance de l'individuel n'est pas d'ailleurs le seul fait de l'homme : le Dieu d'Aristote ne connaît pas non plus les choses individuelles, lesquelles ne procèdent pas de Lui. L'intelligibilité du singulier lui est à jamais impossible. Le singulier est inintelligible pour Dieu et pour l'homme : il l'est donc en soi.

n'est pas le cas de l'optique pascalienne : toute chose, fût-elle singulière, dès qu'elle est, est intelligible parce que créée. Elle possède une signification, sinon pour l'homme, du moins pour Dieu : «L'auteur de ces merveilles les comprend » (fr. 230). C'est pourquoi l'auteur des Pensées peut vouloir comprendre ce qui fait l'individualité de chaque personne car Dieu, Lui, la connaît. S'il ne le peut, ce n'est pas en raison d'une inintelligibilité foncière de l'individuel, mais bien parce que sa capacité d'intellection est limitée: l'homme ne peut rendre compte ni de l'infiniment petit, ni de l'infiniment grand: il en est de même pour l'individualité du moi. S'il existe bien une opacité du singulier pour Pascal, celle-ci souligne moins l'inintelligibilité de la res singularis que la finitude de la ratio mensurans. Pascal, en cherchant à analyser le singulier, oppose ainsi au primat de l'intelligence défendu par Aristote, le primat du réel.

Ces deux conceptions divergentes de la nature de l'être et de son intelligibilité impliquent également une conceptualisation différente de la vérité. Le philosophe aristotélicien face à l'univocité de l'être, objet naturel de l'intelligence humaine, organise toute sa réflexion autour du principe de non-contradiction :

Il est impossible que le même attribut appartienne et n'appartienne pas en même temps au même sujet et sous le même rapport ${ }^{71}$.

Il définit ce principe comme une « ultime vérité, car il est par nature un point de départ, même pour les autres axiomes ${ }^{72}$ ». Celui-ci souligne l'étroite dépendance du vrai à l'être, car penser vrai signifie très exactement penser séparé ce qui est séparé, et uni ce qui est uni. Aristote souligne ici que l'univocité de l'être implique l'univocité du vrai : il note ainsi, dans la Métaphysique, que pour les choses qui ne sauraient être autrement qu'elles sont :

la même opinion ne devient pas tantôt vraie et tantôt fausse, mais les mêmes opinions sont éternellement vraies ou fausses ${ }^{73}$. 
71 La pérennité de l'être implique celle du vrai et la connaissance humaine peut ainsi acquérir des certitudes. Pascal maintient cette unité très forte qui existe entre la nature de l'être et celle du vrai, mais l'être étant pour lui une notion dialectique, la vérité se doit de posséder la même caractéristique. Loin d'être évidente, celle-ci est connue le plus souvent de façon indirecte: c'est par l'impossibilité de sa contradictoire que la raison rend raison d'une vérité. Il existe ainsi une connaissance par négation. Les concepts que nous possédons ne sont pas la vérité mais plutôt les erreurs opposées entre lesquels se meut la vérité, " bornes entre lesquelles passe la voie qui mène au vrai ${ }^{74}$ ". La vérité ne pourra, ainsi, être obtenue qu'au prix d'un sacrifice continuel de nos opinions, bornées par nature : Pascal souligne lui-même que la fausseté n'est souvent que l'exclusion d'une vérité.

Tous errent d'autant plus dangereusement qu'ils suivent chacun une vérité : leur faute n'est pas de suivre une fausseté, mais de ne pas suivre une autre vérité. (fr. 690)

72 Mais dès lors, cet art d'unir les contraires, de rendre conciliable ce qui semblait exclusif l'un de l'autre, est un art proprement divin qui demande une lumière supérieure, car il faut une pensée qui n'ait pas à réduire les choses pour les comprendre mais qui les voit chacune à sa place, dans leur unité et diversité :

Tous leurs principes sont vrais, des pyrrhoniens, des stoïques, des athées etc. Mais

leurs conclusions sont fausses, parce que les principes opposés sont vrais aussi.

(fr. 512)

ceci explique l'importance de la notion de perspective chez Pascal. L'homme ne peut jamais élaborer un système, qui enferme la réalité dans son ordre particulier mais il doit se contenter d'une méthode : il ne peut embrasser l'ordre qui régit l'univers, mais doit en revanche s'acheminer vers lui. La raison humaine pose, en effet, elle-même des contradictions dont la solution lui échappe. De fait, pour Pascal, ce n'est qu'en JésusChrist que l'on comprend la véritable perspective de chaque chose: "En Jésus-Christ toutes les contradictions sont accordées»(fr. 289). Hors de lui, toute connaissance humaine demeure caduque, transitoire. L'appréhension de la vérité, pour l'homme, demeure fondamentalement indirecte.

Or, Pascal emploie un terme particulier pour désigner ce qui n'est ni la vérité ni le mensonge, mais cet entre-deux qui est proprement ce que l'homme connaît naturellement, quand lui manque la vision surnaturelle de la foi : c'est le terme de figure.

Tout ce qui ne va point à l'unique bien en est la figure. Car puisqu'il n'y a qu'un but, tout ce qui n'y va point en mots propres est figure. (fr. 301)

74 La figure n'est pas la vérité mais au regard de celui qui la nie comme réalité, elle pourra désigner la vérité. Prendre la figure pour la fin reviendrait à ne saisir du chiffre que le signe apparent ; considérer la figure comme ce qu'elle est, requiert qu'on la dépasse pour aller vers ce dont elle est le négatif, l'ordre de la grâce et de la charité. Ainsi, le concept de nature, dont nous avons considéré le caractère dialectique, peut être dit la figure de l'ordre de la charité. Comme le note P. Magnard, « la place tenue par la nature dans l'édifice apologétique devrait maintenant nous apparaître: premier degré dans l'ascension, elle doit cependant être niée en son ordre pour devenir figure de l'ordre supérieur ${ }^{75}$ ». Toute la réalité peut être ainsi analysée en termes de vérité ou de figure de la vérité ${ }^{76}$. L'homme possède ainsi un mode d'appréhension du réel et du vrai fondamentalement indirect : pour connaître le réel, il doit s'astreindre à un travail de 
sélection des faits qui fonctionne comme un préalable indispensable; pour atteindre le vrai, il doit auparavant déchiffrer les signes qui le figurent.

Si, chez Aristote, ni le concept de « raison des effets » ni celui de figure n'apparaissent, le Stagirite avait cependant, bien avant Pascal, insisté sur cette discursivité de la raison humaine, et ce, à travers son étude de l'analogie. Distinguant les concepts univoques, équivoques et analogues, il définit ce dernier comme un concept portant sur des réalités essentiellement diverses mais ayant, cependant, entre elles un certain rapport. Intermédiaire entre l'univoque et l'équivoque, il désigne une notion qui s'applique à plusieurs sujets en un sens ni totalement identique ni totalement différent. Dans le premier chapitre des Topiques, Aristote l'utilise pour résoudre la question de la nature. Pour ce faire, il distingue trois principes : le sujet et les deux contraires, lettré et illettré par exemple. Le sujet est ce qui permet aux deux contraires de communiquer, et sans être un contraire de plus, est autre chose que les deux contraires. Or, de quelle manière le sujet, le substrat est-il pensable? Aristote note qu'il l'est par analogie : ce que l'airain est à la statue, le sujet l'est à la substance. L'analogie est ainsi une ressemblance de rapport qui n'exclut pas une différence. Comportant en elle-même une affirmation et une négation, elle relève, elle aussi, d'un processus dialectique. Ce qui caractérise ainsi l'analogie aristotélicienne est la médiété : l'esprit humain y a, en effet, recours à chaque fois qu'il ne peut appréhender une réalité directement. Nous sommes bien proches alors de la figure pascalienne, qui désigne, elle aussi, une réalité de façon médiate. La différence entre l'analogie aristotélicienne et la figure pascalienne est que la première est un moyen d'approche particulier qui souligne la déficience de la raison à appréhender tel ou tel objet, alors que la seconde est l'unique façon qu'a l'homme de chercher à connaître toute réalité, quelle qu'elle soit.

\section{IV. - La morale}

Si Pascal conteste l'indépendance de la métaphysique à l'égard de la théologie, a fortiori le fera-t-il pour ce qui se réfère à l'éthique. De ce point de vue, le disciple de Port-Royal semble n'avoir que peu de rapports avec le Stagirite. Pascal est, avant tout, un chrétien qui espère la conversion de ses lecteurs. Aussi s'oppose-t-il d'emblée à toute morale dite "des philosophes", c'est-à-dire à toute morale établie par la simple raison humaine, objet même de l'Éthique à Nicomaque :

Mais la vertu qui doit faire l'objet de notre examen est évidemment une vertu humaine puisque le bien que nous cherchons est un bien humain et le bonheur un bonheur humain ${ }^{77}$.

77 Le point de départ est cependant commun: constater une recherche universelle de bonheur. Pascal écrit: "Tous les hommes recherchent d'être heureux. Cela est sans exception.» (fr. 181) ; Aristote, qui tente de déterminer le bien auquel tendent tous les hommes, affirme :

Sur son nom en tous cas, la plupart des hommes sont pratiquement d'accord : c'est le bonheur ${ }^{78}$.

Le Stagirite a alors grand soin de faire prendre conscience à son lecteur de la difficulté de l'analyse et il insiste sur la complexité des réalités morales et sociales qui ne requièrent pas le même type d'exigence que les mathématiques. Le caractère changeant du jugement des hommes sur le bonheur ou sur le bien peut dérouter, voire décourager, celui qui cherche quelques principes stables. Pascal note dans l'Art de persuader que : 
La raison de cette extrême difficulté vient de ce que les principes de plaisir ne sont pas fermes et stables. Ils sont divers en tous les hommes, et variables dans chaque particulier ${ }^{79} \ldots$ à sa matière : non pas la méthode déductive mais diaporématique, qui consiste à mettre en présence une thèse et une antithèse défendables, à en dégager les éléments de vérité qu'elles contiennent et à prendre pour principe, en vue de l'établissement de la science morale, la définition acquise de cette façon. Cependant, cette méthode contient un présupposé que ne peut accepter Pascal : en effet, un tel choix implique que les opinions humaines (thèse et antithèse défendables) contiennent toujours une certaine part de vérité ; or, Pascal insiste fondamentalement sur l'impuissance naturelle de l'homme à atteindre le vrai. Si pour Aristote, c'est la raison qui peut décréter la droite règle, l'ỏ $\ominus$ ò $\lambda$ $\lambda$ үo en matière morale, Pascal se montre, lui, infiniment plus méfiant :

Toutes vos lumières ne peuvent arriver qu'à connaître que ce n'est point dans vousmêmes que vous trouverez ni la vérité ni le bien. Les philosophes vous l'ont promis et ils n'ont pu le faire. Ils ne savent ni quel est votre véritable bien, ni quel est votre véritable état. (fr. 182)

81 Cette divergence sur la méthode à observer recouvre un désaccord plus profond, qui porte sur le concept même de nature humaine et sur ses implications en matière morale. Ils affirment, en effet, tous deux que l'homme cherche le bonheur et l'acquiert par la

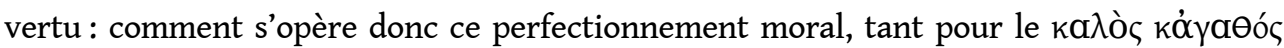
que pour l'« honnête homme »? Aristote se montre très clair dans l'Éthique à Nicomaque : il pose l'existence d'une base stable, la nature humaine, composée d'âme et de corps; il analyse ensuite l'âme de la façon suivante: il distingue trois sortes d'état, qui sont

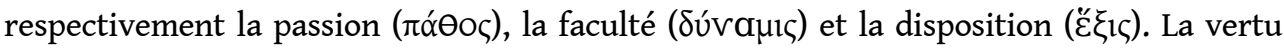
ne peut être une passion car la crainte ne saurait être dite vertueuse, ni une faculté, car celle-ci peut se mettre au service du mal comme du bien; elle ne peut donc être qu'une disposition, un habitus. Certes, toute disposition n'est pas vertu, mais la vertu est une disposition résultant d'une délibération volontaire. Ainsi pour que l'acte soit vraiment moral, il faut que celui qui agisse le fasse dans certaines conditions :

En premier lieu, il doit savoir ce qu'il fait; ensuite choisir librement l'acte en question et le choisir en vue de cet acte lui-même ; et en troisième lieu, l'accomplir dans une disposition d'esprit ferme et inébranlable ${ }^{81}$.

Aristote développe ici une distinction capitale entre l'aptitude, la manière d'être, puissance déjà déterminée, et l'ह̌ $\zeta ı$ qui en est l'exercice. Cette distinction a été retenue par les philosophes scolastiques sous la désignation de actus primus/actus secundus ou matière première/ matière seconde, cette dernière étant la nature morale acquise à force de répétition d'actes. Cette distinction s'avère, en effet, décisive à l'heure de déterminer le rôle et le champ d'application de la liberté humaine : l'ensemble des ह̌ notre caractère, notre personnalité morale. Dès lors se pose une seconde question : $\mathrm{s}^{\prime} \mathrm{il}$ est de notre ressort d'acquérir de bons habitus, discerner leur bonté ou leur malice l'est-il 
également? Aristote reprend-il à son compte l'assertion socratique : «Nul n'est méchant volontairement »? Il répond au livre III :

La poursuite de la fin n'est pas ainsi l'objet d'un choix personnel, mais exige qu'on soit né, pour ainsi dire, avec un œil qui nous permettra de juger sainement et de choisir le bien véritable ${ }^{82}$.

Sans admettre une doctrine qui professerait que tout est une question de tempérament et que le bien nous est inné, Aristote est donc loin de repousser la nécessité, dans la

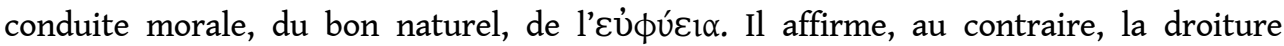
naturelle de l'homme ainsi que son attirance innée vers le bien : un homme foncièrement méchant n'aurait, en effet, aucun désir d'acquérir la vertu. Certes, cette attirance première vers le bien se trouve contrariée à l'intérieur même de l'homme ; Aristote fait état de cette lutte :

Mais il se manifeste aussi en eux un autre principe, qui se trouve par sa nature même, en dehors du principe raisonnable, principe avec lequel il est en conflit et auquel il oppose de la résistance ${ }^{83}$.

Il reconnaît ainsi que la bonté morale s'obtient au prix de nombreux efforts : cependant, ce principe de résistance apparaît parfaitement maîtrisable. L'homme doit ainsi devenir bon, et surtout, il peut devenir tel. Qu'en est-il de Pascal ?

À maintes reprises dans les Pensées, Pascal souligne l'importance de la coutume : est-ce là une autre manière de désigner l'habitude aristotélicienne, acquise à force de répétitions d'actes? Le fragment 159 est, à ce propos, fort explicite : si Aristote présentait les habitus comme des dispositions à acquérir par la mise en pratique, Pascal souligne, lui, que la coutume n'est pas de l'ordre de l'agir mais bien de l'être.

La coutume est une seconde nature, qui détruit la première. Mais qu'est-ce que nature? Pourquoi la coutume n'est-elle pas naturelle? J'ai grand peur que cette nature ne soit elle-même qu'une première coutume, comme la coutume est une seconde nature. (fr. 159)

La distinction entre nature et coutume, entre l'inné et l'acquis, si claire chez le Stagirite, s'annule chez l'auteur des Pensées ${ }^{84}$. La nature humaine, blessée par le péché originel, ne saurait trouver, sans la grâce, un principe lui permettant de refaire son unité. Le seul principe qui demeure est celui de la coutume, ou de manière plus concrète, celui de l'automate, de la machine, qui relève de l'ordre du corps. La coutume s'enracine, en effet, dans la dimension matérielle de l'homme en tant qu'il est situé dans l'espace et dans le temps. Nous avions déjà noté, en physique aussi bien qu'en métaphysique, la scission qu'opérait Pascal entre l'instinct et la raison, entre la matière et l'esprit. Nous devons en considérer maintenant les implications morales. Cette conception, héritée du mécanisme, implique une réduction, voire une négation de la liberté humaine; en effet, un tel principe fournit à Pascal une source fictive de légitimité, tant pour les vertus acquises de manière individuelle que pour l'autorité et la justice, bases de la société.

87 S'instaure ainsi une vision de l'homme radicalement différente de celle proposée par Aristote dans La Politique. L'homme y est décrit comme capable de bien, doué d'une nature jouant le rôle de norme, et surtout défini comme « animal politique » :

Nous sommes créés en vue de la vie en société : car nous avons un sentiment du juste et de l'injuste qui ne sert que dans la vie en société, et la nature ne fait rien en vain $^{85}$.

De fait, pour Aristote, le tout est antérieur à la partie : la cité est par là-même antérieure, du point de vue chronologique et éthique, au citoyen. Celle-ci connait la véritable justice 
car la loi y est définie comme une décision de la raison pure, «libre de tout désir ». Aristote élabore ainsi une véritable philosophie politique, qui souligne le fait que l'individu ne s'épanouit pleinement qu'au sein des différentes communautés que sont respectivement la famille, le village et la cité, répondant au célèbre verset de l'Iliade, symbole pour les Grecs de toutes les malédictions : être « sans famille, sans loi, sans foyer ${ }^{86} »$.

Pour Pascal, en revanche, la société n'est qu'un agrégat d'individus foncièrement égoïstes : « Tous les hommes se haïssent naturellement l'un l'autre » (fr. 243). Égoïsme qui implique que si la société existe, c'est en tant que fruit de la coutume et non de la nature. Dès lors, la justice humaine pour Pascal est un leurre : dans sa lutte avec la force, elle a perdu son propre fondement et gagné celui que lui confère la coutume :

Et ainsi ne pouvant faire que ce qui est juste fût fort, on a fait que ce qui est fort fût juste. (fr. 135)

Alors qu'Aristote opère continuellement la distinction, dans son ouvrage, entre l'homme bon et l'homme pervers, Pascal ne mentionne que l'égocentrisme de la nature humaine. Le combat mentionné par Aristote entre un principe raisonnable et un principe déraisonnable, entre la droite raison et l'instinct aveugle au sein d'une nature saine, devient, chez Pascal, une guerre entre une raison déficiente et des passions très fortes, au sein d'une nature pourrie. L'auteur des Pensées note qu'après le péché originel,

La concupiscence s'est donc élevée dans ses membres et a chatouillé et délecté sa volonté dans le mal, et les ténèbres ont rempli son esprit de telle sorte que sa volonté, auparavant indifférente pour le bien et pour le mal (...) se trouve maintenant charmée par la concupiscence qui s'est élevée dans ses membres. Et son esprit très fort, très juste, très éclairé, est obscurci et dans l'ignorance ${ }^{87}$.

91 L'issue du combat est ainsi non seulement prévisible mais inéluctable; seule la grâce pourra rendre quelque équilibre à cette lutte. Alors qu'Aristote ne pouvait que constater et chercher un remède, Pascal, par le biais de la Révélation, explique le mécanisme de cette lutte ; mais une telle explication relève de la foi, puisqu'elle prend en compte une analyse de la nature humaine faite à la lumière de la Révélation. La morale de Pascal ne peut s'élaborer en marge du christianisme.

Pascal a conservé, cependant, les concepts clefs de l'éthique aristotélicienne; les définitions de la vertu et du mal nous le prouvent. Aristote écrit au chapitre II de l'Éthique à Nicomaque :

La vertu est une sorte de médiété en ce qu'elle vise le moyen ${ }^{88}$.

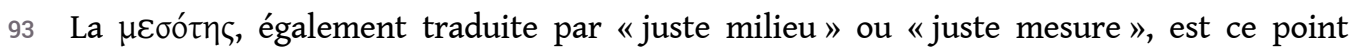
d'équilibre entre deux facteurs rivaux, établi en fonction de nous-mêmes; Aristote insiste sur le fait qu'il s'agit d'un «moyen par rapport à nous ${ }^{89}$ ». Mais s'il est vrai que la vertu soit une médiété, une mesure, il n'y a en elle, cependant, aucune mesure ni excès.

La mesure qui définit la vertu et la fait être elle-même n'est pas une mesure d'ellemême, mais une mesure de l'activité dont elle est le degré mesurés ${ }^{\circ}$.

94 Cette notion de «juste milieu» ne joue pas simplement un rôle dans la morale aristotélicienne : son origine est, encore une fois, métaphysique. En effet, selon Aristote, les éléments eux-mêmes, du fait qu'ils comportent du plus et du moins dans le chaud, le froid, le lourd et le léger, et que sont ainsi détruits les excès réciproques de ces qualités contraires, sont des exemples de mixtes. Cela suppose la nécessité, pour les contraires, d'avoir atteint un moyen terme dans lequel leur opposition perd ce qu'elle avait 
d'extrême et de radical, et s'exprime finalement dans une relation qui est un ópӨò $\varsigma$ 入óyos.

Elle connaît également des implications en logique : la découverte d'un moyen terme permet au syllogisme de lier dans un jugement un sujet et un attribut, lesquels ont un fond commun mais dont l'un a en trop ce que l'autre a en moins : « mortel » se dit de tous les vivants et de tous les hommes; tandis que du sujet Socrate, cette double universalité est absente. Ce «juste milieu » entre deux excès, loin d'être une médiocrité, est une excellence. Le courage est ainsi le juste milieu entre la peur et la témérité ; la tempérance,

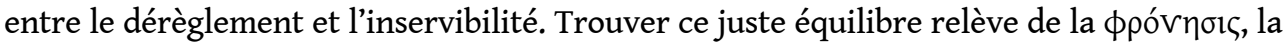
prudence aristotélicienne.

Pascal, dans les Pensées, parvient à la même conclusion :

Rien que la médiocrité n'est bon (...) C'est sortir de l'humanité que de sortir du milieu. La grandeur de l'âme humaine consiste à savoir s'y tenir. Tant s'en faut que la grandeur soit à en sortir, qu'elle est à n'en point sortir. (fr. 452)

Pour l'un comme pour l'autre, la vertu est liée à la notion d'équilibre et de limite : elle concerne proprement la limite humaine. L'homme doit rester à la place qui lui est dévolue.

En creux, s'élabore une conception du mal comme transgression d'un point, d'un milieu, d'une limite. Pour les Grecs, le plus grand des maux est l'v̀bpıc, cet excès né du désir de l'homme de se faire l'égal ou le rival des dieux. Pour Pascal, le véritable mal est le péché originel, péché d'orgueil s'il en est, commis sous l'injonction de Satan: "Vos yeux s'ouvriront et vous serez comme des dieux. » $(\mathrm{Gn}, 1,3)$. Ce premier péché est à la racine de tous les autres; c'est pourquoi pour Pascal comme pour Aristote, il ne faut pas chercher à sortir de son ordre :

Car tout tend à soi : cela est contre tout ordre. Il faut tendre au général, et la pente vers soi est le commencement de tout désordre. (fr. 680)

9 Les notions de bien et de mal s'élaborent donc conjointement à celle de nature humaine, car pour l'un et l'autre, le mal est vu comme la privation d'un bien et d'un bien qui est dû. Pour Aristote, en cela disciple des Pythagoriciens, le mal est l'illimité, le désordre même :

De plus l'erreur est multiforme (car le mal relève de l'Illimité, et le bien du Limité); tandis qu'on ne peut observer la droite règle que d'une seule façon (...) Et c'est ce qui fait que le vice a pour caractéristiques l'excès et le défaut, et la vertu la médiété :

L'honnêteté n'a qu'une forme, mais le vice en a de nombreuses ${ }^{91}$.

Pascal ne dit pas autre chose :

Le mal est aisé, il y en a une infinité, le bien presque unique. (fr. 454)

1 Ainsi, divergeant quant à l'optique fondamentale de leur morale, l'un s'appuyant uniquement sur la nature, l'autre principalement sur la grâce, Pascal et Aristote manient, une fois de plus de façon identique, les concepts clefs de ce champ philosophique, ceux du vice et de la vertu.

Ce rapide parcours nous permet de formuler, en conclusion, une double remarque : la première concerne l'aspect novateur de la pensée de Pascal. Ce dernier a pu, en effet, occulter le très riche héritage dont celui-ci a fait l'objet: nous voudrions souligner qu'à l'égard de l'auteur des Pensées, un tel jugement mérite d'être nuancé; et, en ce sens, retrouver des filiations, loin d'altérer la nouveauté de sa démarche, procure à celle-ci, nous semble-t-il, une solide assise philosophique. 
La seconde concerne le rapport de Pascal à la philosophie traditionnelle : l'augustinisme pascalien se conjugue, ici, avec une vision du monde et de l'homme qui réutilise les notions aristotéliciennes d'ordre, de hiérarchie et de juste milieu. À un platonisme avoué : «Platon, pour disposer au christianisme» (fr. 505), vient s'ajouter un aristotélisme caché ; loin de mépriser la science des philosophes, Pascal, pour exposer son propos et convertir son libertin, n’a donc rejeté aucune de leurs écoles.

\section{NOTES}

1. L'Entretien avec M. de Sacy, in Euvres, Paris, éd. de J. Mesnard, Paris, Desclée de Brouwer, 1991, t. III, p. 129. Toutes nos références à la correspondance et aux différents opuscules de Pascal seront faites dans cette édition; pour les Pensées, elles le seront dans celle de Ph. Sellier, Paris, Bordas, coll. Garnier Flammarion, 1991.

2. Comme le souligne Jean Mesnard dans son introduction à l'opuscule, il convient de « replacer [celui-ci] dans la tradition aristotélicienne ». Euvres, tome III, Desclée de Brouwer, 1991, p. 377.

3. La Logique ou l'art de penser, Paris, éd. Flammarion, 1970, Premier Discours, p. 41.

4. Sur les rapports entre l'opuscule de Pascal et l'ouvrage d'Arnauld et Nicole, voir la notice du texte de Pascal dans l'édition de J. Mesnard au tome III (p. 360 à 368); consulter également celle de l'édition Le Guern, second tome de la Pléiade, 2000, (p. 1177 et sq.) ainsi que, dans le même volume, celle précédant la Contribution à la Logique de Port-Royal, p. 1170 à 1173.

5. L'Esprit géométrique, in Cuvres, t. III, p. 390.

6. Seconds Analytiques, II, 8, 93a.

7. Lettre à Le Pailleur, in Euvres, t. II, p. 562-563.

8. Seconds Analytiques, II, 10, 93b.

9. Métaphysique, Z, 12, $1037 \mathrm{~b}$.

10. Ibid.

11. L'Esprit géométrique, op. cit., p. 396.

12. Louis Marin souligne que si elle l'atteint, elle ne saurait alors l'enserrer : «La fonction du signe mot est alors d'arrêter cette fuite du signifié en l'enveloppant par l'unité d'une forme signifiante. » La Critique du discours. Sur La Logique de Port-Royal et les Pensées de Pascal, éd. de Minuit, 1975, p. 124.

13. Ibid., p. 398.

14. Lettre à Le Pailleur, op. cit., p. 563.

15. «Sont des principes propres, par exemple, les définitions de la ligne et du droit. » Seconds Analytiques, II, 8, 93a.

16. Descartes, Discours de la méthode, éd. Adam-Tannery, 1996, $\mathrm{II}^{\mathrm{e}}$ partie, p. 18.

17. L'Esprit géométrique, op. cit., p. 393.

18. «Car, quand elle est arrivée aux premières vérités connues, [la science géométrique] s'arrête là et demande qu'on les accorde, n'ayant rien de plus clair pour les prouver. » L'Esprit géométrique, op. cit., p. 400.

19. Ibid., p. 395.

20. Seconds Analytiques, I, 10, 76a.

21. L'Esprit géométrique, op. cit., p. 395.

22. Seconds Analytiques, I, 10, 76a. 
23. Cf. Éthique à Nicomaque, VI, 7, 1141a.

24. L'Esprit géométrique, op. cit., p. 395.

25. J. Prigent, « Pascal pyrrhonien, géomètre, chrétien », in Pascal présent, Textes du tricentenaire 1662-1962, éd. Fayard, coll. Écrivains d'Auvergne, 1963, p. 64.

26. « Cœur.

Instinct.

Principes. » (fr. 187)

27. Seconds Analytiques, II, 19, $100 \mathrm{~b}$.

28. Ces deux formes d'esprit relèvent bien, toutes deux, de la raison discursive dans la mesure où chacune renvoie à une manière particulière de considérer ce qui est démonstratif.

29. J. Laporte, «L'Apologétique », in Pascal Présent, p. 59.

30. «Ces termes-là désignent si naturellement les choses qu'ils signifient, à ceux qui entendent la langue, que l'éclaircissement qu'on en voudrait faire apporterait plus d'obscurité que d'instruction. » L'Esprit géométrique, op. cit., p. 396.

31. « De l'Art de persuader ", in L'Esprit géométrique, op. cit., p. 415.

32. Topiques, I, ch. 2, 101a.

33. « De l'Art de persuader », in L'Esprit géométrique, op. cit., p. 415-416.

34. Ibid., p. 415.

35. Ibid., p. 413.

36. Voir aussi plus récemment l'ouvrage de Rémi Brague, La Sagesse du monde, Fayard, 1999, ch. XII « La fin d'un monde », p. 213 et sq.

37. Physique, I, 1, 184a.

38. Lettre au Père Noël, in Euvres, t. II, p. 519.

39. Récit de la grande expérience de l'équilibre des liqueurs, in Euvres, t. II, p. 689.

40. Traité de l'équilibre des liqueurs, in Euvres, t. II, p. 1060.

41. Nous renvoyons ici à Dominique Descotes, L'Argumentation chez Pascal, P.U.F., 1993, III partie, ch. 7, p. 166 et sq.

42. Le Père Noël est, au dire même de Pascal, un parfait représentant de la pensée d'Aristote : «On ne peut [lui] refuser la gloire d'avoir soutenu la physique péripatéticienne aussi bien qu'il est possible de le faire. » Lettre au Père Noël, op. cit., p. 527.

43. Donnée en Physique, IV, 212a.

44. Lettre à Le Pailleur, op. cit., p. 566.

45. Ibid.

46. Ibid., p. 564-565.

47. Lettre du Père Noël, op. cit., p. 529.

48. Physique, IV, 1, 208b.

49. Lettre du Père Noël, op. cit., p. 530.

50. Cf. Physique, III, 8,208 .

51. Physique, III, 8, 206b.

52. Nous renvoyons ici à l'analyse qu'en donne Pierre Magnard, «L'infini pascalien », Revue de l'enseignement philosophique, $\mathrm{XXXI}^{\mathrm{e}}$ année, pp. 2-16.

53. C'est pourquoi l'exclamation « Le silence éternel de ces espaces infinis m'effraie » (fr. 233) est bien à attribuer au libertin et non à Pascal lui-même.

54. Sur le passage de l'une à l'autre, voir les pages très éclairantes de M. Serres, Le Système de Leibniz et ses modèles mathématiques, Paris, P.U.F., 1968, p. 700 et sq.

55. Métaphysique, D, 9, $1074 \mathrm{~b}$.

56. Physique, VIII, 5, $285 \mathrm{~b}$.

57. De la Génération et de la Corruption, I, 6, 323a.

58. Sur cette omission que P. Magnard appelle à juste titre «l'esquive de l'origine », voir La Clé du chiffre, Paris, éditions universitaires, 1991, IIe partie, ch. 1, p. 155 à 163. 
59. «La grandeur de l'homme est si visible qu'elle se tire même de sa misère. Car ce qui est nature aux animaux, nous l'appelons misère en l'homme. » (fr. 149).

60. J. Mesnard, "Universalité de Pascal », in Méthodes chez Pascal, Actes du colloque tenu à Clermont-Ferrand, P.U.F, 1976, p. 335-356.

61. Éthique à Nicomaque, I, 6, 1098a.

62. Nous ne pouvons que renvoyer ici à l'analyse qu'en donne Vincent Carraud dans Pascal et la philosophie, P.U.F., 1992, p. 113 et sq.

63. Métaphysique, $\mathrm{Z}, 17,1041 \mathrm{~b}$.

64. Physique, II, 1, $19 \mathrm{~b}$.

65. Métaphysique, L, 3, 1070a. C'est ici que passe, pour Aristote, la frontière entre le naturel et l'artificiel.

66. Pierre Magnard, « Pascal dialecticien », in Pascal présent, p. 260.

67. Laurent Thirouin, «La Raison des effets. Essai d'explication d'un concept pascalien », XVII siècle, janv. mars $82, \mathrm{n}^{\circ} 134$, p. 48.

68. Ibid., p. 36.

69. Jean Molino, «La Raison des effets », in Méthodes chez Pascal, p. 493.

70. En réalité, il y en a pour Aristote : ce sont les astres. Mais il n'en est pas de même pour les choses sensibles, pour celles du moins qui constituent le monde sublunaire. cf. Métaphysique, M, 3, 1078a.

71. Métaphysique, G, 3, $1005 \mathrm{~b}$.

72. Ibid.

73. Métaphysique, Q, 10, $1051 \mathrm{~b}$.

74. J. Chevalier, Cadences, éd. Plon, 1951, t. II, ch. 1, p. 53.

75. P. Magnard, La Clé du chiffre, $\mathrm{I}^{\mathrm{re}}$ partie, ch. 3, p. 122.

76. «La figure a été faite sur la vérité, et la vérité a été reconnue sur la figure. » (fr. 667)

77. Éthique à Nicomaque, I, 13, 1102a.

78. Ibid., I, 2, 1095a.

79. « De l'Art de persuader », in L'Esprit géométrique, op. cit., p. 417.

80. Op. cit., I, 1, 1094 b.

81. Ibid., II, 3, 1105a.

82. Ibid., III, 7, 1114b.

83. Ibid., I, 13, $1102 b$.

84. Sur la question des rapports entre nature et coutume, voir Gérard Ferreyrolles, Les Reines du monde, Champion, 1995, p. 61 et sq.

85. La Politique, I, 1, 1253a.

86. Iliade, IX, 63.

87. Écrits sur la grâce, in Euvres, t. III, p. 793-794.

88. Éthique à Nicomaque, II, 5, 1106b.

89. Ibid.

90. Ibid.

91. Ibid., II, 5, 1106b. 
INDEX

Mots-clés : Pascal, Aristote, philosophie, logique, physique, métaphysique, morale

Keywords : Pascal, Aristotle, philosophy, logic, physics, metaphysics, moral

\section{AUTEUR}

HÉLÈNE MICHON

Centre d'Études Supérieures de la Renaissance, Université François Rabelais, Tours. 